International Journal of Latest Trends in Engineering and Technology

Vol.(8)Issue(1), pp.551-557

DOI: http://dx.doi.org/10.21172/1.81.072

e-ISSN:2278-621X

\title{
A DATA ANALYTICS PROTOTYPE FOR HANDLING SOFTWARE BUG TRIAGE PROCEDURES
}

\author{
LV Srinivas ${ }^{1}$, R Shiva Shankar ${ }^{2}, \mathrm{~K}$ Venkateswara Rao ${ }^{3}$
}

\begin{abstract}
Bug triage is an imperative stride during the time spent bug settling. The objective of bug triage is to allot another coming bug to the right potential designer. The vast majority of the software companies need to manage substantial number of programming bugs consistently. Programming bugs are unavoidable and settling programming bugs is a costly errand. The objective of compelling bug triaging programming is to allocate conceivably experienced engineers to new-coming bug reports. In this paper we analyze different data reduction techniques on the accuracy of bug assignment. We process experiments on real time data sets, by utilizing mining strategies, mining programming storehouses can reveal fascinating data in programming archives and take care of certifiable programming issue like Eclipse, Mozilla and GNOME. In experimental evaluation we evaluate data reduction for bug triage on bug reports on open source tools like Eclipse, Mozilla and so on.

Index Terms: Bug Triage, Data Reduction in Bug Report, Preprocessing the Bug Report, Fixing Bugs,

Application of Data Preprocessing, Data M anagement in Bug Repositories, Bug Data Reduction.
\end{abstract}

\section{INTRODUCTION}

Software data bases (Repository) include significant data about programming tasks. This data can help to deal with the advancement of these undertakings. In the most recent decade, specialists have examined and mined these product stores to bolster programming improvement and advancement [1]. A bug archive assumes a critical part in overseeing programming bugs. Numerous open source programming tasks have an open bug store that permits both engineers and clients to submit abandons or issues in the product, propose conceivable improvements, and remark on existing bug reports. Many programming organizations spend the vast majority of the cash in altering the bugs. Extensive programming tasks have bug storehouse that gathers all the data identified with bugs.

In bug archive, every product bug has a bug report. The bug report comprises of printed data with respect to the bug what's more, overhauls identified with status of bug altering. When a bug report is framed, a human triage allocates this bug to a designer, who will attempt to settle this bug. This designer is recorded in a thing allotted to. The appointed to will change to another designer if the already relegated designer can't settle this bug. The procedure of allotting a right engineer for settling the bug is called bug triage. Bug triage is a standout amongst the most tedious stride in treatment of bugs in programming tasks. Manual bug triage by a human triage is tedious furthermore, blunder inclined subsequent to the quantity of day by day bugs is vast and absence of learning in designers about all

\footnotetext{
${ }^{1}$ Department of CSE, SRKR Engineering College, Bhimavaram, AP

${ }^{2}$ Department of CSE, SRKR Engineering College, Bhimavaram, AP

${ }^{3}$ Developer in IT \& Technical Consultant, Computer Science Department, JNTU University, AP
} 
bugs [3][4]. On account of every one of these things, bug triage results in lavish time misfortune, high cost and low precision. The data put away in bug reports has two primary difficulties. Firstly the vast scale information and furthermore low nature of information. Because of vast number of day by day reported bugs, the number of bug reports is scaling up in the store. Uproarious and excess bugs are debasing the nature of bug reports.

In this paper we analyze five different effective bug triage methodologies in data reduction for processing software projects. Which will diminish the bug information to spare the work expense of engineers? It additionally intends to manufacture a removing so as to astound arrangement of bug information the repetitive and non-educational bug reports. We consolidate diverse methods of occurrence choice and highlight determination to all the while lessen the bug measurement and the word measurement [6]. The diminished bug information contain less bug reports and less words than the first bug information and give comparative data over the first bug information. Test results demonstrate that five strategies to the information set can diminish bug reports yet the exactness of bug triage may be diminished; applying the element determination system can decrease words in the bug information and the precision can be expanded.

The remainder of this paper organizes as follows: Section II describes motivation of bug triage in real time software projects. Section III presents Literature review process in software bug triage evaluation. Section IV, V, VI, VII describes different data reduction techniques in real time software product development. Section VIII describes concluding remarks of bug triage via open source software's.

\section{MOTIVATION OF BUG TRIAGE IN DATA REDUCTION}

In the bug storehouses, all the bug reports are filled by the designers in regular dialects. The low quality bugs gather in bug stores with the development in scale [7].

\section{Think of some as cases:}

We list the bug report of bug 205900 of Eclipse in Case 1 (the portrayal in the bug report is in part precluded) to think about the expressions of bug reports.

a) Case 1 (Bug 205900) Current form in Eclipse Europe disclosure store broken. [Plug-ins] all introduced accurately and don't demonstrate any slips in Plug-in arrangement view. At whatever point I attempt to include a [diagram name] outline, the wizard can't be begun because of a missing [class name] class [8][9]. In this bug report, a few words, e.g., introduced, appear, began, and missing, are generally utilized for portraying bugs. For content order, such normal words are not useful for the nature of expectation. Thus, we tend to uproot these words to lessen the calculation for bug triage. Then again, for the content characterization, the repetitive words in bugs can't be evacuated specifically. Subsequently, we need to adjust an important system for bug triage.

Case 2 (Bug 201598). 3.3.1 About says 3.3.0. Assemble id: M20070829-0800. 3.3.1 About says 3.3.0.)

This bug report shows the lapse in the adaptation dialog. Be that as it may, the points of interest are not clear. Unless an engineer is extremely acquainted with the foundation of this bug, it is elusive the points of interest. As indicated by the thing history, this bug is altered by the designer who has reported this bug. Be that as it may, the outline of this bug may make different engineers befuddled.

Also, from the point of view of information preparing, particularly programmed handling, the words in this bug may be uprooted following these words are not useful to recognize this bug. In this manner, it is important to evacuate the loud bug reports and words for bug triage.

Case 3. Bugs 200019 and 204653.

(Bug 200019) Argument popup not highlighting the right contention ...(Bug 204653) Argument highlighting wrong. In [10] bug stores, the bug report of bug 200019 is stamped as a copy one of bug 204653 (a copy bug report, indicates that a bug report portrays one programming deficiency, which has the same main driver as a current bug report). The printed substance of these two bug reports is comparable. Subsequently, one of these two bug reports may be picked as the agent one. In this manner, we need to utilize a sure strategy to uproot one of these bug reports.

In light of the above three cases, it is important to propose a way to deal with lessening the scale (e.g., extensive scale words in Case 1) and enlarging the nature of bug information (e.g., loud bug reports in Case 2) and repetitive bug reports in Case 3).

\section{LITERATURE REVIEW PROCESS IN SOFTWARE BUG TRIAGE}

In [1] they specify that Bug triaging is a slip inclined, dull and tedious assignment. They are running with Revisiting Bug Triage and Resolution Practices. In this paper they learned about bug triaging and settling works on, including bug reassignments and re-openings, in the connection of the Mozilla Core and Firefox ventures, which they consider to be illustrative samples of an expansive scale open source programming undertaking. Likewise they have plan to lead subjective and quantitative examination of the bug task rehearses. We are keen on giving bits of knowledge into 
a few territories: triage practices, audit and endorsement procedures; main driver investigation of bug reassignments and revives in open source programming undertakings; and proposals for enhancements/upgrade of bug following frameworks.

In [2] this paper, they present a diagram model taking into account Markov chains, which catches bug hurling history. This model has a few alluring qualities. To begin with, it uncovers designer systems which can be utilized to find group structures and to discover suitable specialists for another assignment. Second, it serves to better relegate engineers to bug reports. In our investigations with 445,000 bug reports, our model decreased hurling occasions, by up to $72 \%$. Moreover, the model expanded the expectation exactness by up to 23 rate focuses contrasted with customary bug triaging methodologies.

In [3] late research demonstrates that upgrading suggestion exactness issue and proposes an answer that is basically an occasion of Content Based Retrieval (CBR). Then again, CBR is surely understood to bring about overspecialization, suggesting just the sorts of bugs that every engineer has settled some time recently. This issue is basic by and by, as some accomplished engineers could be over-burden, and this would moderate the bug settling procedure. In this paper to start with, we reformulate the issue as a streamlining issue of both precision and expense. Second, we receive a substance Content Boosted Collaborative Filtering (CBCF), consolidating a current CBR with a Collaborative Filtering recommender $(\mathrm{CF})$, which upgrades the proposal nature of either approach alone.

In [4] Current systems either utilize data recovery and machine figuring out how to locate the most comparable bugs as of now settled and suggest master engineers, or they break down change data originating from source code to propose master bug solvers. Neither one of the techniques consolidates printed similitude with change set examination and along these lines misuses the inter linking's capability between bug reports and change sets. Studies have demonstrated that viable bug triaging is done cooperatively in a meeting, as it requires the coordination of various people, the venture's comprehension setting and the particular's comprehension work hones.

\section{FEATURE WITH INSTANCE SELECTION BASED SOFTWARE TRIAGE}

We introduce our preparation set decrease approach for bug triage. In our work, an element choice calculation and an occasion choice calculation are joined to decrease loud or repetitive data in the preparation set of bug triage.

We utilize both component determination and case choice calculations to evacuate the superfluous elements and examples. Drawn on the involvement in content classification, a component in bug triage shows a word while an occasion demonstrates a bug report [9]. In this manner, the objective of our work is to diminish the content lattice of the preparation set on two measurements, i.e., the word measurement and the bug report measurement. To create a decreased preparing set on two measurements, we utilize a two-stage mix to exploit both element choice and occurrence determination (a stage is a procedure applying one calculation). The lessened preparing set is connected to supplant the first preparing arrangement of bug triage. To recognize the blends, we research the two's request stages. Given an element determination calculation FS and an occasion choice calculation IS, we utilize FS $\rightarrow$ IS to signify first applying FS and afterward IS. Then again, IS $\rightarrow$ FS means first applying IS and after that FS.

Input: preparing set T with $\mathrm{n}$ words and $\mathrm{m}$ bug
reports, highlight determination calculation FS ,
last number of words F n , example choice
calculation IS , last number of bug reports I m ,
request of the blend FS $\rightarrow$ IS.
Output: lessened preparing set T FI for bug triage
1) Apply FS to n expressions of T and ascertain
target values for every one of the words;
2) Select the top F n expressions of T and produce
a preparation set T F ;
3) Apply IS to m bug reports of T F ;
4) Terminate IS the point at which the quantity of
bug reports is equivalent or short of what I m and
produce the last preparing set T FI.

For the mixtures of FS and IS, we will present the outcomes of FS $\rightarrow$ IS and IS $\rightarrow$ FS, respectively.

Highlight determination is a standard innovation to lessen the elements of huge scale information sets in machine learning. Since numerous component choice calculations have been examined for content classification, we select an 
average calculation in our work, i.e., $\chi 2$ - test (CHI) 3 [11][12]. Yang \& Pedersen have given a relative study on five component determination calculations and have reported that $\mathrm{CHI}$ can outflank alternate calculations in the study. $\mathrm{CHI}$ is a regular component determination calculation, which measures the reliance in the middle of words and engineers.

\section{AUTOMATIC APPROACH FOR DATA REDUCTION}

The outline in figure 1 showed the framework structural planning of the proposed framework. The information to the framework is as bug information set. The bug information set comprises all the subtle elements of programming bugs. Every bug has bug report and the subtle elements of the engineer who have chipped away at that individual bug. The bug report is primarily partitioned into two sections, outline and betrayal [14]. The proposed framework gives anticipated results in type of yield. Fundamentally, there are two sorts of clients in the proposed framework. To begin with is the designer furthermore, second is the analyzer. Designer will get programming bugs relegated to him. Engineer can chip away at one and only programming bug at once. Analyzer can add new bugs to the framework.

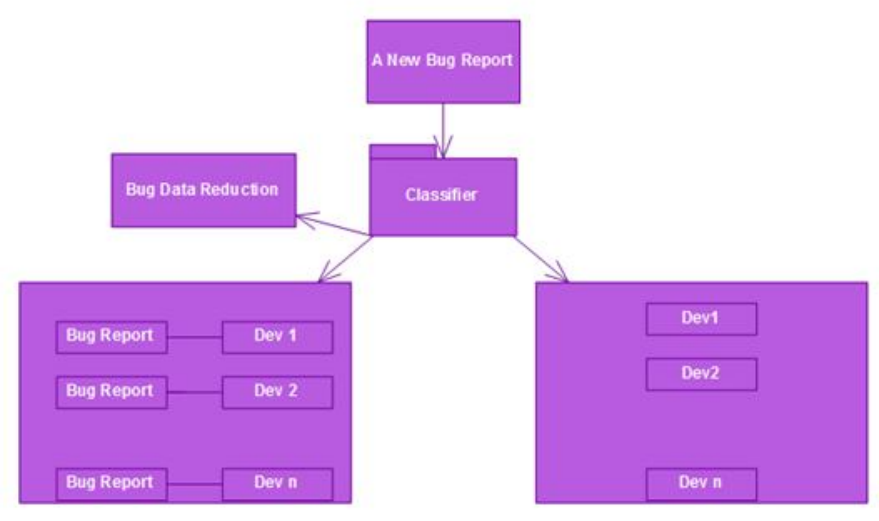

Figure 1: Automatic bug tracking appearance in real time software product assurance.

As appeared in figure 1, the proposed framework makes utilization of bug information lessening. In the proposed framework, to spare the work expense of designers, the information lessening for bug triage is made. Bug daya lessening is connected in period of information arrangement of bug triage. Information lessening for the most part has two objectives [13]. Firstly, diminishing the information scale and also, enhancing the precision of bug triage. Methods of occasion determination and highlight choice are utilized for information decrease. Occasion determination and highlight choice are broadly utilized methods as a part of information handling. For a given information set in a sure application, case choice is to acquire a subset of significant occasions (i.e., bug reports in bug information) while highlight choice means to get a subset of pertinent elements (i.e., words in bug information). In the proposed framework, the blend of occurrence determination and highlight determination is utilized.

The proposed framework will be executed in java dialect so it will be stage free. As there is no confinement on the extent of bug's data, an analyzer can include vast number of bugs in the framework [15]. This is one of the greatest preferences of the proposed framework. Since the entire bug's data is interested in every one of the engineers, it sets aside less time for the designer to take the choice. Designer can rapidly pick the bug to settle.

Since bug triage intends to foresee the engineers who can settle the bugs, we take after the current work to evacuate unfixed bug reports, e.g., the new bug reports or will-not-alter bug reports [16]. Hence, we just pick bug reports, which are settled what's more, copy (taking into account the things status of bug reports). In addition, in bug vaults, a few designers have just altered not very many bugs. Such inert engineers may not give adequate data to anticipating right designers.

\section{SELECTION APPROACH FOR DATA REDUCTION}

To decrease the time spent triaging, we display a methodology for programmed triaging by prescribing one experienced designer for each new bug report. Our methodology utilizes a machine learning calculation to suggest an engineer who may be fitting for determining the bug. We plan the bug triaging procedure as a characterization 
assignment where cases speak to bug reports, elements speak to the report's terms, and the class mark speaks to the engineer who settled this report [16]. This methodology can help the triage process in two ways: 1) it may permit a triage to process a bug all the more rapidly, and 2) it may permit a triage with less information about frameworks and designers to perform bug assignments all the more precisely. Our methodology obliges a task to have had an open bug storehouse for some time frame in which the examples of who illuminates what sorts of bugs can be found out.

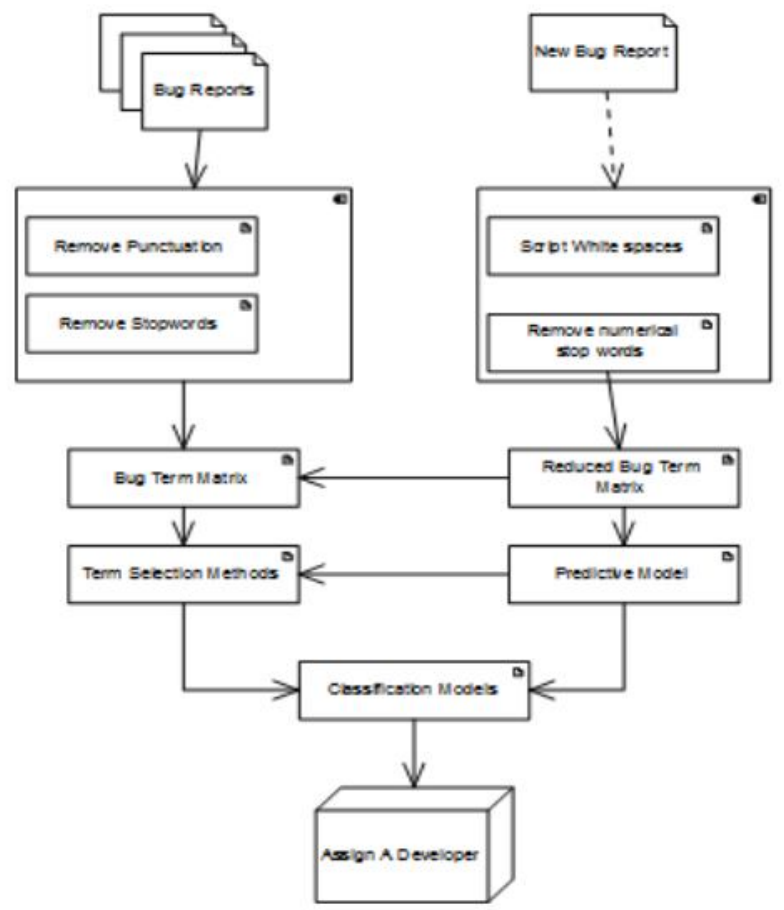

Figure 2: Bug selection procedure for testing of software product assurance.

Fig. 2 demonstrates an abnormal state portrayal of our proposed approach. Bug reports are unstructured information which may contain superfluous words. In this manner, we apply the customary content handling way to deal with change the content information into a significant representation. We utilize the synopsis of bug reports as a depiction of bugs [17][18]. The content handling incorporates white-spaces, accentuation, numbers, and stop words evacuation. After that, the methodology builds a bug-term network weighted by term recurrence. At that point, distinctive term choice routines are connected to diminish both the dimensional and the meager condition of information. The following step is to assemble a classifier utilizing the Naive Bayes approach. The classifier is prepared utilizing the preparation information set (bug reports).

\section{EFFECTIVE BUG TRIAGE WITH DATA REDUCTION PROCESS}

In this section we analyze and combine both feature selection and instance selection attribute on uploaded data sets. As shown in above algorithm, we propose bug information lessening to diminish the scale and to enhance the nature of information in bug repositories. We first present how to apply occurrence determination and highlight choice to bug information, i.e., information decrease for bug triage. At that point, we list the information's advantage decrease. In bug triage, bug information set is changed over into a content network with two measurements, in particular the bug measurement and the word measurement [17]. In our work, we influence the mix of occurrence determination and highlight choice to produce lessened bug information set. We supplant the first information set with the diminished information set for bug triage.

Case choice and highlight choice are broadly utilized systems as a part of information preparing. For a given information set in a sure application, example choice is to get a subset of significant occasions (i.e., bug reports in bug information) while highlight determination intends to acquire a subset of important components (i.e., words in bug information). In our work, we utilize the blend of occasion determination and highlight choice. An Instance Feature determination calculation IS and a component choice calculation FS, FS ! IS and IS ! FS are seen as two 
requests for applying decreasing methods [9]. Subsequently, a test is the way to focus the request of lessening methods, i.e., how to pick one between FS! IS and IS! FS. We allude to this issue as the expectation for decrease orders. To apply the information decrease to each new bug information set, we have to check the exactness of both two requests (FS! IS and IS! FS) and pick a superior one. To keep away from the time expense of physically checking both lessening requests, we consider anticipating the decrease request for another bug information set in light of authentic information sets [19][20].

To apply the information decrease to each new bug information set, we have to check the precision of both two requests (FS! IS and IS! FS) and pick a superior one. To keep away from the time expense of physically checking both decrease orders, we consider foreseeing the diminishment request for another bug information set taking into account recorded information sets.

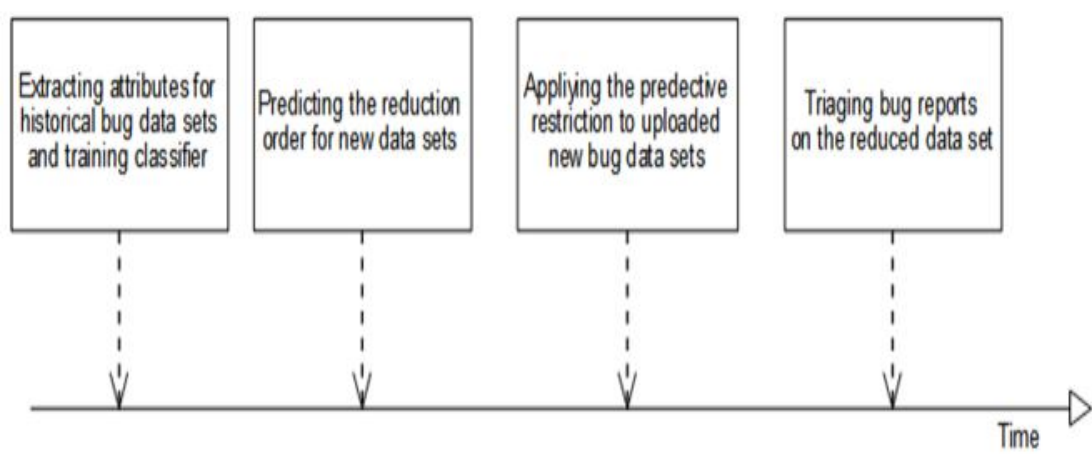

Figure 3: Procedure of predictive reduction orders for bug triage.

Bug information set is mapped to an occasion and the related lessening request (either FS! IS or IS! FS) is mapped to the mark of a class of occurrences. Fig. 3 outlines the progressions of anticipating lessening requests for bug triage. Note that a classifier can be prepared just once when confronting numerous new bug information sets. That is, preparing such a classifier once can foresee the decrease orders for all the new information sets without checking both diminishment orders [21]. To date, the issue of foreseeing lessening requests of applying element choice and occasion determination has not been explored in other application situations.

From the point of view of programming designing, anticipating the decrease request for bug information sets can be seen as a sort of programming measurements, which includes exercises for measuring some property for a bit of programming. On the other hand, the components in our work are extricated from the bug information set while the elements in existing take a shot at programming measurements are for individual programming artifacts, 3 e.g., an individual bug report or an individual bit of code. In this paper, to keep away from questionable meanings, a property alludes to a separated component of bug information set while an element alludes to an expression of a bug report.

\section{CONCLUSION}

Bug triage is an extravagant stride of programming support in both work cost and time cost. In this paper, we consolidate highlight choice with occasion choice to diminish the size of bug information sets and additionally enhance the information quality. To focus the request of applying occurrence choice and highlight determination for another bug information set, we concentrate qualities of every bug information set and prepare a prescient model taking into account chronicled information sets. We exactly research the information diminishment for bug triage in bug vaults of two expansive open source ventures, to be specific Eclipse and Mozilla. Our work gives a way to deal with utilizing procedures on information preparing to shape lessened and excellent bug information in programming improvement and support.

\section{REFERENCES}

[1] Revisiting Bug Triage and Resolution Practices, Olga Baysal, Reid Holmes, and Michael W. Godfrey David R. Chariton School of Computer Science University of Waterloo, ON, Canada \{obaysal, rtholmes, migod\}@uwaterloo.ca.

[2] Improving Bug Triage with Bug Tossing Graphs Gaeul Jeong * Seoul National University gejeong@ropas.snu.ac.kr. 
[3] COSTRIAGE: A Cost-Aware Triage Algorithm for Bug Reporting Systems Jin-woo Park, Mu-Woong Lee, Jinhan Kim, Seung-won Hwang, POSTECH, Korea, Rublic jwpark85,sigliel,wlsgks08,swhwang\} @ postech.edu

[4] Collaborative Bug Triaging using Textual Similarities and Change Set Analysis, Katja Kevic, Sebastian C. Muller, Thomas Fritz, and Harald C. Gall '. Department of Informatics University of Zurich, Switzerland katja.kevic@uzh.ch \{smueller, fritz, gall\}@ifi.uzh.ch.

[5] "Towards Effective Bug Triage with Software Data Reduction Techniques", by Jifeng Xuan, He Jiang proceedings in IEEE TRANSACTIONS ON KNOWLEDGE AND DATA ENGINEERING, VOL. 27, NO. 1, JANUARY 2015.

[6]" Efficient Bug Triaging Using Text Mining”, by Mamdouh Alenezi and Kenneth Magel, proceedings in @ 2013 ACADEMY PUBLISHER.

[7]" Bug Triage with Bug Data Reduction"by Pankaj Gakare1, Yogita Dhole2, Sara Anjum, proceedings in International Research Journal of Engineering and Technology (IRJET)Volume: 02 Issue: 04 | July-2015.

[8] “Analysis of Bug Triage using Data Preprocessing (Reduction) Techniques" by G. Parthasarathy,

D.C. Tomar, International Journal of Computer Applications (0975 - 8887) Volume 125 - No.9, September 2015.

[9]" A Survey on Software Data Reduction Techniques for Effective Bug Triage" by Ashwini Jadhav1, Komal Jadhav2, Anuja Bhalerao3, Amol Kharade, proceedings in Ashwini Jadhav et al, / (IJCSIT) International Journal of Computer Science and Information Technologies, Vol. 6 (5), 2015, 4611-4612.

[10] "Finding Bug by using Data Reduced Techniques" by Amruta Gadekar1 , Pranjali Taralkar 2, Nikita Waghmare3, Rahul Dapke, proceedings in Gadekar et al, / (IJCSIT) International Journal of Computer Science and Information Technologies, Vol. 6 (5), 2015, 4263-4265.

[11] "Towards Training Set Reduction for Bug Triage" by Weiqin Zou Yan Hu, Jifeng Xuan, proceedings in Joint Meeting European Software Engineering Conf. \& ACM SIGSOFT Symp. Foundations of Software Engineering (ESEC-FSE 09), ACM, Aug. 2009, pp. 111-120.

[12] D. _Cubrani_c and G. C. Murphy, “Automatic bug triage using text categorization," in Proc. 16th Int. Conf. Softw. Eng. Knowl. Eng., Jun. 2004, pp. 92-97.

[13] Eclipse. (2014). [Online]. Available: http://eclipse.org/

[14] B. Fitzgerald, "The transformation of open source software," MIS Quart., vol. 30, no. 3, pp. 587-598, Sep. 2006.

[15] A. K. Farahat, A. Ghodsi, M. S. Kamel, "Efficient greedy feature selection for unsupervised learning," Knowl. Inform. Syst., vol. 35, no. 2, pp. 285-310, May 2013.

[16] N. E. Fenton and S. L. Pfleeger, Software Metrics: A Rigorous and Practical Approach, 2nd ed. Boston, MA, USA: PWS Publishing, 1998.

[17] Y. Freund and R. E. Schapire, "Experiments with a new boosting algorithm," in Proc. 13th Int. Conf. Mach. Learn., Jul. 1996, pp. 148- 156.

[18] Y. Fu, X. Zhu, and B. Li, “A survey on instance selection for active learning,” Knowl. Inform. Syst., vol. 35, no. 2, pp. 249-283, 2013.

[19] I. Guyon and A. Elisseeff, “An introduction to variable and feature selection,” J. Mach. Learn. Res., vol. 3, pp. 1157-1182, 2003.

[20] M. Grochowski and N. Jankowski, "Comparison of instance selection algorithms ii, results and comments," in Proc. 7th Int. Conf. Artif. Intell. Softw. Comput., Jun. 2004, pp. 580-585.

[21] K. Gao, T. M. Khoshgoftaar, and A. Napolitano, "Impact of data sampling on stability of feature selection for software measurement data," in Proc. 23rd IEEE Int. Conf. Tools Artif. Intell., Nov. 2011, pp. 1004-1011. 